\title{
Fully-covered esophageal stent migration rates in benign and malignant disease: a multicenter retrospective study
}

\section{다)(1) $(\odot$}

\author{
Authors \\ Samuel Thomas ${ }^{1}$, Ali A. Siddiqui ${ }^{2}$, Linda Jo Taylor ${ }^{1}$, Sheeva Parbhu ${ }^{1}$, Christopher Cao ${ }^{2}$, David Loren², Thomas \\ Kowalski², Douglas G. Adler ${ }^{1}$
}

Institutions

1 Department of Gastroenterology and Hepatology, University of Utah - Huntsman Cancer Center, Salt Lake City, Utah, United States

2 Department of Gastroenterology and Hepatology, Thomas Jefferson University Hospitals, Philadelphia, Pennsylvania, United States

submitted: 26.10 .2018

accepted after revision: 26.2.2019

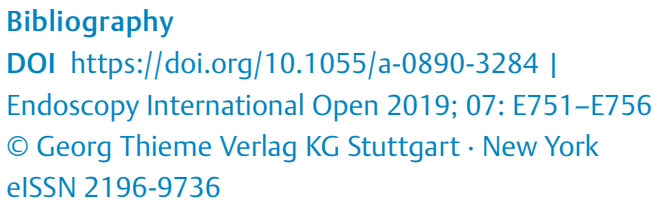

Corresponding author

Douglas G. Adler, MD, FACG, AGAF, FASGE, Professor of Medicine, Director of Therapeutic Endoscopy, Director, GI Fellowship Program, Gastroenterology and Hepatology, University of Utah School of Medicine, Huntsman Cancer Center, 30N 1900E 4R118, Salt Lake City, Utah 84132

Fax: +1-801-581-8007

Douglas.adler@hsc.utah.edu

\section{ABSTRACT}

Background and study aims Stent migration is a common complication of fully-covered self-expanding metal stents (FCSEMS), but the rate of clinically relevant migration as defined by stent migration followed by reintervention via endoscopy for stent replacement is unknown. The goal of this study is to gain insight into the total migration rate and clinically relevant migration rate of different types of FCSEMS placed within benign and malignant strictures with specific attention paid to stent manufacturer, diameter, and length.

Patients and methods Multicenter retrospective analysis of endoscopic data from patients with FCSEMS placed within benign or malignant strictures. FCSEMS used included a variety of sizes and manufacturers.

Results A total of 369 patients were included, 161 of whom had benign strictures and 208 of whom had malignant strictures. The total migration rate and clinically relevant migration rate in benign strictures were $30 \%$ and $17 \%$, respectively. For benign strictures, Wallflex stents had a clinically relevant migration rate of $15 \%$, compared to Endomaxx stents with $19 \%$, and Evolution stents with $25 \%$ $(P=0.52)$. The total migration rate and clinically relevant migration rates in malignant strictures were $23 \%$ and $14 \%$, respectively. Evolution stents had a significantly higher clinically relevant migration rate $(29 \%)$ than the Wallflex stents (7\%) and the endomaxx stents (12\%), $P=0.003$.

Conclusion This study is the largest to investigate migration rates for FCSEMS in benign and malignant strictures. Clinically relevant migration is a relatively common occurrence with all stent types studied and better anti-migration features are needed.

\section{Introduction}

Esophageal fully-covered self-expanding metal stents (FCSEMS) are widely used for treatment of benign and malignant esophageal disorders, including strictures, perforations, anastomotic leaks, and fistulas [1]. Malignant dysphagia secondary to strictures has been an indication for esophageal stenting since the 1960 s [2,3]. Benign dysphagia as a consequence of refractory benign esophageal strictures is a relatively novel indication for esophageal stenting once bougie or balloon dilation has been attempted and failed [4,5]. Major limitations of esophageal stent placement include stent migration, stent occlusion, perforation, hemorrhage, and chest pain. Use of FCSEMS has become popular due to the ability of these stents to be removed at, essentially, any time after placement [6]. 
One of the major criticisms of FCSEMS is their relatively high risk of migration. Previous data include a meta-analysis of 18 studies with a total of 444 patients, which found stent migration occurred in $29 \%$ of patients with benign strictures [7]. Other studies have found higher rates of stent migration in benign strictures ranging from $31 \%$ to $40 \%$ [8-11]. Stents placed in malignant strictures were found in various studies to have migration rates from $23 \%$ to $31 \%[6,12,13]$.

In some clinical scenarios, stent migration may be a beneficial finding, as it may reflect resolution of benign strictures or a positive response of malignant strictures to treatments such as chemoradiation therapy [6]. In other situations, presence of migration may be an undesirable complication, and in these settings, the stent migration itself often represents a failure of stent therapy when the underlying stricture, be it benign or malignant, is still of clinical concern.

Replacement of a stent is indicated based on the degree of migration as well as clinical symptoms (i.e. recurrence of dysphagia, pain, etc) and thereby an inability for the stent to perform its intended function, which we define as clinically relevant migration. The aim of this study was to gain insight into the total migration rate and clinically relevant migration rate of different types of FCSEMS in both benign and malignant strictures with specific attention paid to stent location, type, diameter, and length.

\section{Patients and methods}

We performed a multicenter, retrospective analysis of endoscopic data querying for all patients with benign or malignant stenosis who underwent placement of esophageal FCSEMS between October 2006 and August 2016. This study was performed under Institutional Review Board approval for collection of data from electronic medical records located at the University of Utah and Huntsman Cancer Center in Salt Lake City, Utah, and Jefferson University School of Medicine in Philadelphia, Pennsylvania.

The esophageal FCSEMS used in the current study included the WallFlex stent (Boston Scientific, Natick, Massachusetts, United States), the ENDOMAXX stent (Merit Medical, South Jordan, Utah, United States), and the Evolution stent (Cook Endoscopy, Winston-Salem, North Carolina, United States). All of these metal stents have a covering that extends for their entire length. All stents were deployed over guidewires and with the aid of fluoroscopy. Stent sizes were based on stricture location, size, length and severity, and the diameter of the proximal and distal esophagus. Endoscopic and fluoroscopic confirmation of adequate stent position was obtained in all patients.

Patients included in this study had follow-up within 4 weeks of initial stent placement, in case of adverse events (AEs) or stent dysfunction and then not until any endoscopic re-intervention or death. All AEs were recorded as long as the stent was in place. Stents placed for benign strictures were typically removed once the stricture had resolved, whereas FCSEMS for malignant strictures were removed prior to or even during surgery. Any stents that were endoscopically clipped or sutured in place were excluded from the study.
- Table 1 Patient demographics and stent characteristics from data collected at the University of Utah, Huntsman Cancer Center and Jefferson University School of Medicine.

\begin{tabular}{|c|c|}
\hline \multicolumn{2}{|l|}{ Patient $(n=369)$} \\
\hline Mean age - no. yrs (range) & $63(21-94)$ \\
\hline Sex-no. (\%) & $\begin{array}{l}\text { Male: } 278(75) \\
\text { Female: } 91(25)\end{array}$ \\
\hline \multicolumn{2}{|l|}{ Indication } \\
\hline Benign stricture - no. (\%) & $161(44)$ \\
\hline Malignant stricture - no. (\%) & $208(56)$ \\
\hline \multicolumn{2}{|l|}{ Stent } \\
\hline \multicolumn{2}{|l|}{ Location of stent-no. (\%) } \\
\hline Proximal esophagus & $39(11)$ \\
\hline Mid-esophagus & $69(19)$ \\
\hline Distal esophagus & $261(70)$ \\
\hline \multicolumn{2}{|l|}{ Stent type - no (\%) } \\
\hline Merit medical & $96(26)$ \\
\hline Boston Scientific & $218(59)$ \\
\hline Cook Medical & $55(15)$ \\
\hline Stent diameter - range (mm) & $18-23$ \\
\hline Stents $\geq 20$ mm - no. (\%) & $188(51)$ \\
\hline Stents < 20 mm - no. (\%) & $181(49)$ \\
\hline Stent length - range (mm) & $70-150$ \\
\hline Stents > $100 \mathrm{~mm}-$ no. $(\%)$ & $188(51)$ \\
\hline Stents $\leq 100 \mathrm{~mm}-$ no. $(\%)$ & $181(48)$ \\
\hline
\end{tabular}

Rates of stent migration between the three manufacturers were evaluated using chi square test. Significant results were further analyzed by performing pairwise comparisons and the Benjamini-Hochberg procedure was used to control the false discovery rate.

\section{Results}

A total of 369 patients were identified and included in the analysis, of whom 278 were male (75\%) and 91 were female (25\%) ( $\triangleright$ Table 1). Mean patient age was 63 years (Range $21-94$ ). Of the patients, 161 (44\%) had esophageal FCSEMS placed secondary to benign strictures, whereas 208 (56\%) had esophageal FCSEMS placed to treat a malignant stricture. Strictures were localized in the proximal esophagus in 39 patients (11\%), the mid-esophagus in 69 patients (19\%), and the distal esophagus in 261 patients (70\%).

The three different esophageal FCSEMS included the Wallflex stent (Boston Scientific), which accounted for 218 (59\%) of the stents placed, the Endomaxx stent (Merit Endotek) with $96(26 \%)$ stents placed, and the Evolution stent (Cook Medical) with $55(15 \%)$ stents placed. 


\begin{tabular}{|c|c|c|c|c|}
\hline Stent location & Total & $\begin{array}{l}\text { Clinic rel } \\
\text { migration }\end{array}$ & $\%$ & \\
\hline Proximal & 28 & 5 & $18 \%$ & \\
\hline Mid & 30 & 5 & $17 \%$ & \\
\hline \multirow[t]{2}{*}{ Distal } & 103 & 18 & $17 \%$ & $P$-value \\
\hline & 161 & 28 & $17 \%$ & 0.99 \\
\hline Stent location & Total & $\begin{array}{c}\text { Any } \\
\text { migration }\end{array}$ & $\%$ & \\
\hline Proximal & 28 & 8 & $29 \%$ & \\
\hline Mid & 30 & 8 & $27 \%$ & \\
\hline \multirow[t]{2}{*}{ Distal } & 103 & 33 & $32 \%$ & $P$-value \\
\hline & 161 & 49 & $30 \%$ & 0.83 \\
\hline
\end{tabular}

- Fig. 1 Results showing the total number of stents placed within benign strictures, the number of stents with clinically relevant migration (defined as stent migration requiring replacement) and any migration, separated by stent location. $P$ values suggest no significant difference between stents placed within benign strictures with clinically relevant migration or any migration and stent locations.

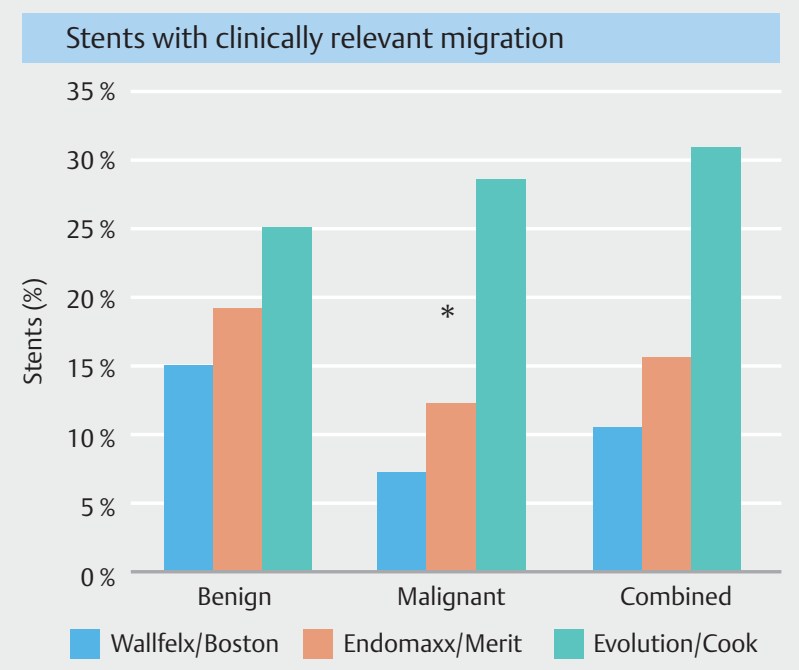

- Fig. 2 The percentage of stents found to have clinically relevant migration (defined as stent migration requiring replacement), separated by stent type and benign vs malignant vs combined benign and malignant strictures. ${ }^{*}$ Indicates the statistically significant difference between the different stent types in relation to malignant strictures.

Stent diameter ranged from 18 to $23 \mathrm{~mm}$. Each manufacturer makes FCSEMS with two different diameters; Cook makes stents that are 18 and $20 \mathrm{~mm}$, Merit makes 19- and 23-mm stents, and Boston Scientific makes 18- and 23-mm stents. For purposes of analysis, stents were grouped by diameter and separated into stents $\geq 20$ or $<20 \mathrm{~mm}$ in diameter. The number of
Bengin strictures and stent type

\begin{tabular}{|c|c|c|c|c|}
\hline Stent type & Total & $\begin{array}{l}\text { Clinic rel } \\
\text { migration }\end{array}$ & $\%$ & \\
\hline Merit & 47 & 9 & $19 \%$ & \\
\hline Boston & 94 & 14 & $15 \%$ & \\
\hline \multirow[t]{2}{*}{ Cook } & 20 & 5 & $25 \%$ & $P$-value \\
\hline & 161 & 28 & $17 \%$ & 0.52 \\
\hline Stent type & Total & $\begin{array}{c}\text { Any } \\
\text { migration }\end{array}$ & $\%$ & \\
\hline Merit & 47 & 19 & $40 \%$ & \\
\hline Boston & 94 & 24 & $26 \%$ & \\
\hline \multirow[t]{2}{*}{ Cook } & 20 & 6 & $30 \%$ & $P$-value \\
\hline & 161 & 49 & $30 \%$ & 0.19 \\
\hline
\end{tabular}

- Fig. 3 Results showing the total number of stents placed within benign strictures, the number of stents with clinically relevant migration (defined as stent migration requiring replacement) and any migration, separated by stent type. $P$ values suggest no significant difference between stents placed within benign strictures with clinically relevant migration or any migration and stent type.

stents $\geq 20 \mathrm{~mm}$ in diameter was 188 (51\%) compared to 181 (49\%) that were $<20 \mathrm{~mm}$.

Stent length ranged from 70 to $150 \mathrm{~mm}$. Each manufacturer makes three or four different lengths of FCSEMS; Cook makes stents that are 80,100 , and $120 \mathrm{~mm}$; Merit makes 70-, 100-, 120-, and 150-mm stents; and Boston Scientific makes 100-, $120-$, and $150-\mathrm{mm}$ stents. For purposes of analysis, stents were grouped by length and separated into stents $>100$ or $\leq 100 \mathrm{~mm}$ in length. The number of stents $>100 \mathrm{~mm}$ in length was $188(51 \%)$ compared to $181(49 \%)$ that were $\leq 100 \mathrm{~mm}$.

\section{Stent migration in patients with benign strictures}

A total of 161 FCSEMS were placed within benign strictures. Our database identified 28 strictures (17\%) in the proximal esophagus, 30 strictures (19\%) in the mid esophagus, and 103 strictures $(64 \%)$ in the distal esophagus. Of the 161 FCSEMS, a total of $32(20 \%)$ were noted to have a late complication that required stent replacement. Twenty-eight of the 32 stents replaced were due to clinically relevant migration. The four remaining stents were replaced due to esophageal perforation, stent occlusion, chest pain and one other non-defined complication.

Of the 28 stents in the proximal esophagus, 5 stents (18\%) had clinically relevant migration and eight (29\%) had any migration, compared to the 30 stents in the mid-esophagus with 5 stents (17\%) with clinically relevant migration and 8 (27\%) with any migration, and lastly compared to the 103 stents in the distal esophagus with 18 stents (17\%) with clinically relevant migration and 33 (32\%) with any migration. We found no significant difference between stents placed within benign strictures with clinically relevant migration or any migration and stent location ( $P=0.99$ and 0.83 respectively) ( $\triangleright$ Fig. 1 ). 


\begin{tabular}{|c|c|c|c|c|}
\hline \multicolumn{5}{|c|}{ Malignant strictures and stent location } \\
\hline Stent location & Total & $\begin{array}{l}\text { Clinic rel } \\
\text { migration }\end{array}$ & $\%$ & \multirow[b]{4}{*}{$P$-value } \\
\hline \multirow{4}{*}{$\begin{array}{l}\text { Proximal } \\
\text { Mid } \\
\text { Distal }\end{array}$} & 11 & 1 & $9 \%$ & \\
\hline & 40 & 5 & $13 \%$ & \\
\hline & 157 & 19 & $12 \%$ & \\
\hline & 208 & 25 & $12 \%$ & 0.95 \\
\hline Stent location & Total & $\begin{array}{c}\text { Any } \\
\text { migration }\end{array}$ & $\%$ & \multirow{5}{*}{$\begin{array}{l}P \text {-value } \\
\mathbf{0 . 1 7}\end{array}$} \\
\hline \multirow{4}{*}{$\begin{array}{l}\text { Proximal } \\
\text { Mid } \\
\text { Distal }\end{array}$} & 11 & 1 & $9 \%$ & \\
\hline & 40 & 6 & $15 \%$ & \\
\hline & 157 & 41 & $26 \%$ & \\
\hline & 208 & 48 & $23 \%$ & \\
\hline
\end{tabular}

- Fig. 4 Results showing the total number of stents placed within malignant strictures, the number of stents with clinically relevant migration (defined as stent migration requiring replacement) and any migration, separated by stent location. $P$ values suggest no significant difference between stents placed within malignant strictures with clinically relevant migration or any migration and stent locations.

The total migration rate of stents in benign strictures that were replaced due to clinically relevant migration was $17 \%$. When comparing the number of stents replaced for clinically relevant migration with the total number of stents placed in benign strictures for each stent type, we found the Wallflex stents (Boston Scientific) were replaced in 14 of the 94 cases (15\%), compared to the Endomaxx stents (Merit) with replacement in nine of the 47 cases (19\%), and finally the Evolution stents (Cook) with replacement in five of the 20 cases $(25 \%)$ ( $\triangleright$ Fig. 2). Although there appears to be a trend towards less clinically relevant migration for the Wallflex stents compared to the Endomaxx stents and Evolution stents, these differences were not statistically significant $(P=0.52)(\triangleright$ Fig. 3$)$.

The total migration rate for all stents in benign strictures regardless of whether the stent was removed was $30 \%$. The total migration rate of each stent type regardless of stent removal was $26 \%$ for the Wallflex stent, $40 \%$ for the Endomaxx stent, and $30 \%$ for the Evolution stent. Similar to the stents replaced for clinically relevant migrations, there appears to be a trend towards less migration for the Wallflex stents compared to the Endomaxx stents and Evolution stents, although these differences were not statistically significant $(P=0.19)$ ( $>$ Fig. 3 ).

When comparing the diameter of stents in patients with clinically relevant migration in benign strictures, 14 of the 66 stents with a diameter $\geq 20 \mathrm{~mm}$ were replaced compared to 14 of the 95 stents with a diameter $<20 \mathrm{~mm}$ ( $21 \%$ vs $15 \%, P=$ $0.29)$.

When comparing the length of stents in patients with clinically relevant migration in benign strictures, 18 of the 85 stents with a length $>100 \mathrm{~mm}$ were replaced compared to 10 of the 76 stents with a length $\leq 100 \mathrm{~mm}(21 \%$ vs $13 \%, P=0.18)$.
Malignant strictures and stent type

\begin{tabular}{|c|c|c|c|c|}
\hline Stent type & Total & $\begin{array}{l}\text { Clinic rel } \\
\text { migration }\end{array}$ & $\%$ & \multirow[b]{4}{*}{$P$-value } \\
\hline \multirow{2}{*}{$\begin{array}{l}\text { Merit } \\
\text { Boston } \\
\text { Cook }\end{array}$} & 49 & 6 & $12 \%$ & \\
\hline & 124 & 9 & $7 \%$ & \\
\hline \multirow[t]{2}{*}{ Cook } & 35 & 10 & $29 \%$ & \\
\hline & 208 & 25 & $12 \%$ & 0.003 \\
\hline Stent type & Total & $\begin{array}{c}\text { Any } \\
\text { migration }\end{array}$ & $\%$ & \multirow[b]{4}{*}{$P$-value } \\
\hline Merit & 49 & 14 & $29 \%$ & \\
\hline Boston & 124 & 21 & $17 \%$ & \\
\hline \multirow[t]{2}{*}{ Cook } & 35 & 13 & $37 \%$ & \\
\hline & 208 & 48 & $23 \%$ & 0.025 \\
\hline
\end{tabular}

- Fig. 5 Results showing the total number of stents placed within malignant strictures, the number of stents with clinically relevant migration (defined as stent migration requiring replacement) and any migration, separated by stent type. $P$ values suggest a significant difference between stents placed within malignant strictures with clinically relevant migration or any migration and stent type.

\section{Migrations in patients with malignant strictures}

A total of 208 FCSEMS were placed for malignant strictures. Our database identified 11 strictures (5\%) in the proximal esophagus, $40(19 \%)$ in the mid esophagus, and 157 strictures (76\%) in the distal esophagus. Of the 208 FCSEMS, a total of 30 (14\%) were noted to have a late complication that required stent replacement. Twenty-five of the 30 stents replaced were due to clinically relevant migration. The five remaining stents were replaced due to stent occlusion $(n=2)$, chest pain $(n=1)$, and other non-defined complications $(n=2)$.

Of the 11 stents in the proximal esophagus, 1 (9\%) had clinically relevant migration and 1 ( $9 \%$ ) had any migration, compared to the 40 stents in the mid-esophagus with five stents (13\%) with clinically relevant migration and $6(15 \%)$ with any migration, and lastly compared to the 157 stents in the distal esophagus with 19 stents (12\%) with clinically relevant migration and 41 (26\%) with any migration. We found no significant difference between stents placed within malignant strictures with clinically relevant migration or any migration and stent location $(P=0.95$ and 0.17 respectively) ( $\triangleright$ Fig. 4$)$.

The total migration rate of stents in malignant strictures that were replaced due to clinically relevant migration was $12 \%$. When comparing the number of stents replaced for clinically relevant migration with the total number stents placed in malignant strictures for each stent type, we found the Wallflex stents were replaced in nine of the 124 cases (7\%), compared to the Endomaxx stents with replacement in six of the 49 cases (12\%), and the Evolution stents with replacement in 10 of the 35 cases (29\%) ( $>$ Fig. 2 ). The Evolution stent had a higher rate of clinically relevant migration when compared to the Wallfelx and Endomaxx stents $(P=0.003)$ ( Fig. 5). 
The total migration rate of all stents in malignant strictures regardless of whether the stent was removed was $23 \%$. The total migration rate for each stent type regardless of stent removal was $17 \%$ for the Wallflex stent, $37 \%$ for the Evolution stent, and $29 \%$ for the Endomaxx stent. The Evolution stent had a significantly higher rate of any migration when compared to the Wallfelx and Endomaxx stents $(P=0.025)$ ( Fig. 5 ).

When comparing the diameter of stents in patients with clinically relevant migration in malignant strictures, nine of the 122 stents with a diameter $\geq 20 \mathrm{~mm}$ were replaced compared to 16 of the 86 stents with a diameter $<20 \mathrm{~mm}$ ( $7 \%$ vs $19 \%$, $P=0.01)$, suggesting that stents with larger diameter, $\geq 20 \mathrm{~mm}$ compared to $<20 \mathrm{~mm}$, were found to have less clinically relevant migration in malignant strictures that was statistically significant.

When comparing the length of stents in patients with clinically relevant migration in malignant strictures, 10 of the 103 stents with a length $>100 \mathrm{~mm}$ were replaced compared to 15 of the 105 stents with a length $\leq 100 \mathrm{~mm}$ (10\% vs $14 \%, P=$ $0.31)$.

\section{Discussion}

Our study found that almost one in three FCSEMS placed for benign strictures migrated (30\%). This is comparable to the $29 \%$ to $40 \%$ migration rate published previously [7-11]. A key finding for our study is that almost one in five FCSEMS placed for benign strictures required reintervention with stent replacement due to clinically relevant migration (17\%). These new data revealing a relatively high rate of clinically significant migration suggest that further investigation should be undertaken to understand and prevent these migrations requiring reinterventions with stent replacement. We attempted to better understand the nature of FCSEMS migration by analyzing each case broken down by stent location, type, diameter and length, which gave us some clarity with limitations as noted below.

Our analysis of benign and malignant indications for FCSEMS and location in relation to both clinically relevant migration and any migration found that there is no statistical difference between the location of the stricture or stent and the rate of clinically relevant migration or any migration.

When comparing the rate of clinically relevant migration within benign strictures using esophageal FCSEMS from three different manufacturers, we found no statistical difference between the different stent types. When comparing the rate of any stent migration within benign strictures using esophageal FCSEMS from three different manufacturers, we again found no statistically significant difference between the different stent types.

We also found that FCSEMS placed in benign strictures were more likely to have clinically relevant migrations requiring replacement if they had a diameter $\geq 20 \mathrm{~mm}(21 \%$ vs $15 \%, P=$ $0.29)$ and/or a length $>100 \mathrm{~mm}(21 \%$ vs $13 \%, P=0.18)$. Although it appears that small-diameter and shorter stents migrate less in benign strictures, there was no difference found to be statistically significant.
With regards to malignant strictures, our study demonstrates that almost one in four stents $(23 \%)$ were found to have migration. This is compared to various studies that note migration rates of $23 \%$ to $31 \%$ for FCSEMS placed within malignant strictures $[6,12,13]$. Another key finding of the study was that $12 \%$ of the stents placed in malignant strictures were found to have clinically relevant migration.

When comparing the three different stent manufacturers with the rate of clinically relevant migration in malignant strictures, we identified that the Evolution stent had a higher rate of clinically relevant migration when compared to the Wallfelx and Endomaxx stents. This was also found to be true for any stent migration within malignant strictures.

With regards to the diameter and length of the FCSEMS placed in malignant strictures, we found more clinically relevant migration if the diameter was $<20 \mathrm{~mm}(19 \%$ vs $7 \%, P=$ 0.01 ) and/or the length was $\leq 100 \mathrm{~mm}$ ( $14 \%$ vs $10 \%, P=0.31$ ). This is the inverse of what was seen in benign strictures. The reasons for these differences are unclear, but may be due to stricture length and geometry as well as underlying esophageal motility.

This study was novel in that it was the first to investigate clinically relevant migration as defined by reintervention via endoscopy for stent replacement. Limitations of this study include the fact that sutured stents were excluded from the data given the variability of how stents are sutured and lack of data available for sutured stents in our database. Also, given that patient follow-up was within 4 weeks, then not until an AE or reintervention, means that the observed rate of stent migration may be an underestimate of the true rate of migration. That is, some patients may have experienced stent migration, but if that did not result in any symptoms and they did not have any further follow-up, then this would have been missed. Additional information that would be beneficial to a future study would include data regarding time to stent removal and identification of patients with malignant strictures who underwent surgery and/or chemo-radiation.

\section{Conclusion}

In conclusion, clinically relevant migration is a relatively common occurrence and the type of stent used for management of malignant structures matters in terms of reducing clinically relevant migration and reintervention. Better anti-migration features need to be designed and incorporated into future FCSEMS.

\section{Competing interests}

Drs. Adler, Siddiqui, Kowalski, and Loren are consultants for BSC. Adler - Consulting for Merit. 


\section{References}

[1] Sharma P, Kozarek R. Role of esophageal stents in benign and malignant diseases. Am J Gastroenterol 2010; 105: $258-273$

[2] Celestin LR. Permanent intubation in inoperable cancer of the oesophagus and cardia. Ann R Coll Surg Engl 1959; 25: 165-70

[3] Provan JL. Use of Celestin tube for palliation of malignant oesophageal obstruction. Thorax 1969; 24: 599-602

[4] van Boeckel PG, Siersema PD. Refractory esophageal strictures: what to do when dilation fails. Curr Treat Options Gastroenterol 2015; 13 : $47-58$

[5] Vermeulen BD, Siersema PD. Esophageal stenting in clinical practice: an overview. Curr Treat Options Gastroenterol 2018; 16: 260-273

[6] Siddiqui AA, Sarkar A, Beltz $S$ et al. Placement of fully covered selfexpandable metal stents in patients with locally advanced esophageal cancer before neoadjuvant therapy. Gastrointest Endosc 2012; 76: $44-51$

[7] Fuccio L, Hassan C, Frazzoni L et al. Clinical outcomes following stent placement in refractory benign esophageal stricture: a systematic review and meta-analysis. Endoscopy 2016; 48: 141-148
[8] Bakken J, Wong Kee Song L et al. Use of a fully covered self-expandable metal stent for the treatment of benign esophageal diseases. Gastrointest Endosc 2010; 72: 712 - 720

[9] Senousy B, Gupte A, Draganov P et al. Fully covered Alimaxx esophageal metal stents in the endoscopic treatment of benign esophageal diseases. Dig Dis Sci 2010; 55: 3399-3403

[10] Eloubeidi M, Talreja J, Lopes T et al. Success and complications associated with placement of fully covered removable self-expandable metal stents for benign esophageal diseases (with videos). Gastrointest Endosc 2011; 73: 673-681

[11] Suzuki T, Siddiqui A, Taylor LJ et al. Clinical outcomes, efficacy, and adverse events in patients undergoing esophageal stent placement for benign indications. J Clin Gastroenterol 2016; 50: 373-378

[12] Martinez JC, Puc MM, Quiros RM. Esophageal stenting in the setting of malignancy. ISRN Gastroenterology 2011; 2011: 719575

[13] Shenfine J, McNamee P, Steen $\mathrm{N}$ et al. A pragmatic randomised controlled trial of the cost-effectiveness of palliative therapies for patients with inoperable oesophageal cancer. Health Tech Assess 2005; 9: $1-121$ 